\title{
Cochrane
Library
}

Cochrane Database of Systematic Reviews

\section{Quality improvement interventions for improving the detection and management of curable sexually transmitted infections in primary care (Protocol)}

Nattabi B, Gudka S, Ward J, Rumbold A

Nattabi B, Gudka S, Ward J, Rumbold A.

Quality improvement interventions for improving the detection and management of curable sexually transmitted infections in primary care. Cochrane Database of Systematic Reviews 2016, Issue 10. Art. No.: CD012374.

DOI: 10.1002/14651858.CD012374.

www.cochranelibrary.com

Quality improvement interventions for improving the detection and management of curable sexually transmitted infections in primary care (Protocol) 
TABLE OF CONTENTS

HEADER . . . . . . . . . . . . . . . . . . . . . . . . . . . . . . . . . 1

ABSTRACT . . . . . . . . . . . . . . . . . . . . . . . . . . . . . . . . . . . . . . . . . . . . .

BACKGROUND . . . . . . . . . . . . . . . . . . . . . . . . . . . . . . . . . . . .

OBJECTIVES . . . . . . . . . . . . . . . . . . . . . . . . . . . . . . . . . . . . . . .

METHODS . . . . . . . . . . . . . . . . . . . . . . . . . . . . . . . . . . . . . . 6

ACKNOWLEDGEMENTS . . . . . . . . . . . . . . . . . . . . . . . . . . . . . . . . . . . . . . . . 11

REFERENCES . . . . . . . . . . . . . . . . . . . . . . . . . . . . . . . . . . . . . . 12

APPENDICES . . . . . . . . . . . . . . . . . . . . . . . . . . . . . . . . . . . . . 15

CONTRIBUTIONS OF AUTHORS . . . . . . . . . . . . . . . . . . . . . . . . . . . . . . . . . . . . 24

DECLARATIONS OF INTEREST . . . . . . . . . . . . . . . . . . . . . . . . . . . . . . . . . . . . . . 24

SOURCES OF SUPPORT . . . . . . . . . . . . . . . . . . . . . . . . . . . . . . . . . .

Quality improvement interventions for improving the detection and management of curable sexually transmitted infections in primary i care (Protocol)

Copyright $\odot 2016$ The Cochrane Collaboration. Published by John Wiley \& Sons, Ltd. 


\title{
Quality improvement interventions for improving the detection and management of curable sexually transmitted infections in primary care
}

\author{
Barbara Nattabi ${ }^{1}$, Sajni Gudka ${ }^{2}$, James Ward ${ }^{3}$, Alice Rumbold ${ }^{4}$ \\ ${ }^{1}$ Western Australia Centre for Rural Health, School of Primary, Aboriginal and Rural Health Care, The University of Western Australia, \\ Geraldton, Australia. ${ }^{2}$ Pharmacy, School of Medicine and Pharmacology, The University of Western Australia, Crawley, Australia. \\ ${ }^{3}$ Infection and Immunity, South Australian Health and Medical Research Institute, Adelaide, Australia. ${ }^{4}$ The Robinson Research \\ Institute, The University of Adelaide, Adelaide, Australia
}

Contact address: Barbara Nattabi, Western Australia Centre for Rural Health, School of Primary, Aboriginal and Rural Health Care, The University of Western Australia, 167 Fitzgerald Street, Geraldton, Western Australia, 6530, Australia. barbara.nattabi@uwa.edu.au.

Editorial group: Cochrane STI Group.

Publication status and date: New, published in Issue 10, 2016.

Citation: Nattabi B, Gudka S, Ward J, Rumbold A. Quality improvement interventions for improving the detection and management of curable sexually transmitted infections in primary care. Cochrane Database of Systematic Reviews 2016, Issue 10. Art. No.: CD012374. DOI: 10.1002/14651858.CD012374.

Copyright (C) 2016 The Cochrane Collaboration. Published by John Wiley \& Sons, Ltd.

\begin{abstract}
A B S T R A C T
This is the protocol for a review and there is no abstract. The objectives are as follows:

To assess the effectiveness and safety of quality improvement interventions for the detection and management of curable sexually transmitted infections in primary care.
\end{abstract}

\section{B A C K G R O U N D}

Sexually transmitted infections (STIs), including chlamydia, syphilis, trichomoniasis, gonorrhoea, herpes simplex virus (HSV), human papilloma virus (HPV), hepatitis B and HIV continue to be a major cause of concern globally including in high-, middle- and low-income countries. The four most common curable STIs worldwide are trichomoniasis (143 million estimated cases a year), chlamydia (131 million), gonorrhoea (78 million) and syphilis (5.6 million) (Newman 2015; WHO 2015). In 2013, chlamydia infection was the most notified infection in the USA with a total of 1,401,906 infections, followed by 333,004 cases of gonorrhoea, 17,375 cases of primary and secondary syphilis and 348 cases of congenital syphilis (CDC 2014). Similarly, chlamy- dia is the highest notified infection in Australia where there were 86,136 cases of chlamydia, 15,786 cases of gonorrhoea and 1999 cases of infectious syphilis in 2014 (Kirby 2015). Europe had a total of 384,555 cases of chlamydia reported from 16 European Union (EU) Member States, followed by 52,995 gonorrhoea cases reported from 28 states and 22,237 syphilis cases from 29 states (ECDC 2015). There is also a high incidence of STIs in developing countries with an estimated 92.6 million cases in the World Health Organization (WHO) African Region, 78.5 million in the WHO South-East Asia Region, and 128.2 million in the WHO Western Pacific Region (WHO 2012).

Trichomoniasis is not a notifiable infection in any country (Poole 2013), but it is the most prevalent STI in the USA affecting 3.7

Quality improvement interventions for improving the detection and management of curable sexually transmitted infections in primary care (Protocol)

Copyright $\odot 2016$ The Cochrane Collaboration. Published by John Wiley \& Sons, Ltd. 
million people (Satterwhite 2013). Trichomonas vaginalis (T vaginalis) prevalence rates vary markedly by population and region, being as high as $50 \%$ in rural clinics in South Africa (Bowden 2000), 16.1\% among rural Indigenous communities in Australia (Garrow 2002) and 7.2\% among disadvantaged urban Indigenous communities in Australia (Panaretto 2006), and as low as $0.4 \%$ at an urban sexual health centre in Sydney, Australia (Uddin 2011). Between 2009 and 2011 there were over 16,000 episodes of trichomoniasis in England. However, this is an underestimate of the general population prevalence because these tests were limited to people attending genitourinary medicine clinics, as asymptomatic people are not routinely screened for $T$ vaginalis infection (Mitchell 2014). Rates of infection were highest in patients of black ancestry (Caribbean and otherwise) with just under half of the clients residing in poor areas of England. Women were 15 times more likely to be diagnosed with the infection compared to men (Mitchell 2014).

STIs are of concern because of the significant morbidity they cause, their long-term impact on reproductive and perinatal health, and their social, economic and psychological impact (WHO 2012), as well as their capacity to facilitate the transmission of HIV infection (Fleming 1999; Ward 2010). STIs particularly cause high levels of morbidity among adolescents, young adults and marginalized and vulnerable communities, with the highest burden of disease among people aged less than 25 years. Sixty eight per cent of chlamydia cases in USA were among young people aged between 15 and 24 years, and rates among black and American Indians/ Alaska Natives were 6.4 and 4 times the rate among white Americans, respectively (CDC 2014). In Europe, 67\% of chlamydia cases and $39 \%$ of gonorrhoea cases were among persons aged from 15 to 24 years (ECDC 2015). In Australia the majority of chlamydia diagnoses $(78 \%)$ and gonorrhoea diagnoses (57\%) were also among 15 to 29 year olds. It is noteworthy that the rates among Indigenous Australians of chlamydia, gonorrhoea and syphilis are 3 times, 18 times and 4 times higher respectively, compared to non-Indigenous Australians (Kirby 2015).

\section{Description of the condition}

Chlamydiae are obligate intracellular bacteria that grow in eukaryotic cells and require resources from the host cells for their metabolism and replication (Peeling 1996). Chlamydia trachomatis Serovars D-K species are associated with acute urethritis, cervicitis, pelvic inflammatory disease (PID), Reiters syndrome, Fitz-Hugh Curtis syndrome, and sequelae in women include ectopic pregnancy, chronic pelvic pain and tubal infertility (Peeling 1996). In males, sequelae include proctitis and epididymo-orchitis. Chlamydia is also associated with adverse outcomes in pregnancy including preterm labor, low birth weight (LBW), neonatal death and premature rupture of membranes (PROM) (Peipert 2003). Infants born to mothers infected with chlamydia can present with oph- thalmia neonatorum or neonatal pneumonia, and other mucous membranes can be infected including the oropharynx, urogenital tract and rectum (CDC 2015).

Females infected with chlamydia may present with a vaginal discharge or bleeding between periods, and males with urethritis and epididymitis (Donovan 2004); however up to $80 \%$ of infections are asymptomatic (Peipert 2003). In females, chlamydia can be isolated from first-catch urine (FCU) and endocervical or vaginal swabs, though FCU is not as sensitive as endocervical or vaginal swabs. In males, FCU or urethral swabs can be used to test for chlamydia (ASHA 2014). Nucleic acid amplification tests (NAAT) are highly sensitive for detecting chlamydia organisms (CDC 2015). Recommended regimens for treatment of chlamydia include azithromycin $1 \mathrm{gm}$ orally as a single dose, or doxycycline $100 \mathrm{mg}$ orally twice a day for 7 days; alternative regimens include erythromycin, levofloxacin or ofloxacin.

Neisseria gonorrhoeae (N. gonorrhoeae) is a Gram-negative intracellular obligate bacterium ( $\mathrm{Ng} 2005)$. Gonococcal infections are associated with cervicitis, urethritis, pharyngitis, proctitis, PID, epididymitis and prostatitis; sequelae include chronic pelvic pain and tubal infertility in women, and urethral stricture in men $(\mathrm{Ng}$ 2005). N. gonorrhoeae is also associated with disseminated infection in adults presenting as cutaneous lesions, tenosynovitis and arthritis (Belkacem 2013). In infants, N. gonorrhoeae is associated with ophthalmia neonatorum and neonatal sepsis, arthritis, meningitis, rhinitis, vaginitis and urethritis (CDC 2015). Clinically, gonococcal infections tend to be more severe than chlamydia; however a large proportion of both females and males can be asymptomatic or undiagnosed (Chandeying 2000; Turner 2002). Specimens for testing for $N$. gonorrhoeae depend on the age, sex and sexual orientation of the patient and include urethral, cervical, vaginal, oropharyngeal, rectal and conjunctival specimens with culture and NAAT tests being highly sensitive in detecting $N$. gonorrhoeae. High levels of antimicrobial resistance in many countries have resulted in a change to recommended regimens for treatment of $N$. gonorrhoeae infections; combination therapy including ceftriaxone $250 \mathrm{mg}$ intramuscular (IM) in a single dose with azithromycin $1 \mathrm{~g}$ orally in a single dose is recommended for uncomplicated gonococcal infections of the cervix, urethra, pharynx and rectum (CDC 2015).

Syphilis, a systemic infection, is caused by Treponema pallidum, a Gram-negative spirochaete bacterium. It is associated with spontaneous abortions, stillbirths, prematurity and congenital syphilis (Genc 2000). The disease is categorised into three stages (primary, secondary and tertiary syphilis) and two latent phases (early and late latent infections) (CDC 2015). As an acquired infection, syphilis first presents as a chancre at the site of inoculation after an incubation period of 3 to 90 days, this constituting the primary stage of syphilis (Genc 2000). The chancre may present on the genitalia, or oral or rectal mucosa, and spontaneously resolves after about six weeks. Secondary syphilis may manifest as skin rash, mucocutaneous lesions or generalised lymphadenopathy and, if un- 
treated, goes into a latent phase (a period of no clinical manifestations). The latent phase may last for several years and can develop into more severe systemic disease with cardiac, skin and skeletal manifestations, this constituting the tertiary stage of syphilis (Genc 2000). Latent infection with syphilis is divided into early latency (one year or less from time of infection) and late latency (more than one year since infection). Any stage of syphilis can be complicated by central nervous system (CNS) involvement, i.e. neurosyphilis, or even ocular manifestations. CNS manifestations include cognitive dysfunction, cranial nerve palsies, meningitis and stroke (CDC 2015).

Syphilis can be diagnosed using lesion exudates and tissue samples from the primary chancre, or serological tests (CDC 2015). A definitive diagnosis of syphilis infection requires at least two serological tests. The traditional algorithm includes screening with a nontreponemal test, e.g. Venereal Disease Research Laboratory (VDRL) or Rapid Plasma Reagin (RPR), followed by a confirmatory treponemal test, e.g. Treponema pallidum haemagglutination assay (TPHA), Treponema pallidum particle agglutination assay (TPPA), fluorescent treponemal antibody absorbed (FTA-ABS) tests, or enzyme immunoassays (EIAs) (PAHO 2015). All stages of syphilis can be treated using parenteral penicillin G, but the dosage, preparation and length of treatment will depend on the stage and manifestations of the disease. In the early phase, the purpose of treatment is to prevent sexual and mother-to-child transmission, but in the latent phase the purpose is to prevent complications and prevent mother-to-child transmission of syphilis (CDC 2015). The recommended regimen for adults with primary, secondary and early latent syphilis is 2.4 million units of IM benzathine penicillin in a single dose, and for those with late latent syphilis or latent syphilis of unknown duration, the regimen is 2.4 million unit IM once a week for 3 weeks. The recommended regimen for tertiary syphilis with normal cerebral spinal fluid (CSF) examination is 3 doses of 2.4 million units of benzathine penicillin G IM at one-week intervals; while the recommended regimen for neurosyphilis and ocular syphilis is aqueous crystalline penicillin G 18 to 24 million units per day administered as 3 to 4 million units intravenously (IV) every 4 hours or continuous infusion, for 10 to 14 days (CDC 2015).

Trichomonas vaginalis ( $T$ vaginalis) is a motile anaerobic flagellated parasite which attaches to vaginal epithelial cells but can also be isolated from the cervix, urethra, upper genital tract and the peritoneum. The parasite is associated with high levels of inflammation in the vaginal and urethral mucosa, tissue damage and long-term sequelae (Coleman 2013). Trichomoniasis is also associated with PID, low birth weight, and preterm delivery (Cotch 1997; Johnston 2008). Trichomoniasis may cause vaginitis, presenting as a foul smelling frothy yellow-green discharge and vulvar itching in females, and as balanitis and urethritis in males (Swygard 2004); however a majority of infections are asymptomatic (Bowden 2000). T vaginalis is transmitted via sexual contact and vertically to infants during vaginal delivery, with an incu- bation period of between 4 and 18 days. The organism can be detected from vaginal, endocervical, urethral, semen and urine specimens using wet mount microscopy, NAAT tests, antigen tests or culture, though NAAT tests are more sensitive than culture. The recommended regimen for treatment of trichomoniasis is $2 \mathrm{~g}$ of metronidazole orally or tinidazole $2 \mathrm{~g}$ orally in a single dose. An alternative regimen is $500 \mathrm{mg}$ of metronidazole orally twice a day for seven days (CDC 2015).

\section{Description of the intervention}

For the purpose of this review, a quality improvement (QI) intervention is defined as "any (health service) intervention aimed at reducing the quality gap for a group of patients representative of those encountered in routine practice" (Shojania 2004). The quality gap is the "difference between healthcare processes or outcomes observed in practice, and those thought to be achievable with the most current and effective professional knowledge, the difference [being] attributable in whole or in part to a deficiency that could be addressed by the healthcare system" (Shojania 2004). Quality improvement processes use systems thinking, data analysis and teams of professionals to bring about better outcomes for patients and improved clinical processes (Plsek 1999). A QI intervention could: be targeted at the implementation of a particular process of care or set of processes to benefit patients with a condition; increase the proportion of patients receiving recommended care; target implementation of a structural or organisational feature; attempt to improve outcomes for a broad group of patients; or target a subset of patients that is typically excluded from clinical research and care (Shojania 2004). The Cochrane Effective Practice and Organisation of Care (EPOC) Group provides a taxonomy of four broad classifications of interventions, namely professional interventions, financial interventions, organisational interventions and regulatory interventions, under which are 50 subcategories (EPOC 2002).

Professional interventions are those that specifically target health professionals and include distribution of educational materials, educational meetings, local consensus processes, educational outreach visits, local opinion leaders, patient-mediated interventions, audit and feedback, reminders, marketing and mass media (EPOC 2002). Educational meetings are one of the most common forms of professional interventions and vary in content, type and number of participants, length, frequency and targeted practices (Forsetlund 2009). Educational meetings consist of small or large group sessions and use a multitude of methods to enhance their effectiveness including vignettes, clinical scenarios, standardised patients and simulation. Educational meetings can be further supported using distance education and self-directed learning (Straus 2013). Distribution of educational materials, including printed or published clinical guidelines, journal articles and monographs, is another well-established, accessible, inexpensive, yet passive, QI professional intervention (Giguere 2012). Educational outreach vis- 
its, also known as academic detailing or public interest detailing, involve the use of trained personnel to deliver educational information to health professionals in their own practice setting, with the intention to change their professional practice (OBrien 2007). Used in conjunction with other interventions, educational outreach visits may include an assessment of barriers to evidencebased practice, tailoring of the intervention to those identified barriers, use of a respected professional to deliver the intervention and feedback on health professionals' clinical practice (Straus 2013). Audit and feedback (AF) is also another commonly used professional intervention albeit with variable results (Ivers 2012). AF is defined as "the development of a summary of some aspect of clinical performance over a specific period of time and subsequent provision of that information to individual practitioners, teams or health care organisations" (Brehaut 2012). AF is based on various theories including Diffusion of Innovations and Social Cognition Theories, Theory of Reasoned Action, Theory of Planned Behaviour (Colquhoun 2013) and Feedback Intervention Theory (Kluger 1996).

Other professional interventions include computer reminders that serve to prompt health professionals as they are engaged in the target activity such as when they are prescribing medicine, documenting the clinical encounter in the medical record or ordering investigations (Shojania 2009). Reminders assist the health professional to recall information that is relevant to the task at hand so that the client receives the best possible care. Local consensus processes involve the inclusion of health providers in decision-making processes and it is believed that a sense of ownership may enhance commitment to change and improvement processes (Nasser 2007). Use of local opinion leaders is based on Social Learning Theory whereby people who are seen as credible, competent and trustworthy are deemed possible change agents through their ability to influence and facilitate health professional behaviour change (Flodgren 2011; Grol 2007). Patient-mediated interventions, which include patient decision aids, communication skills training to patients and professionals, and patients' reports to health professionals, also aim to enhance evidence-based practice among health professionals through patient-provider interactions (EPOC 2002b; Straus 2013). Other professional interventions include mass media, e.g. use of radio, television and newspapers to reach a large number of health professionals, and marketing, which includes surveys and focus groups, targeting a range of providers (EPOC 2002b).

Financial interventions target both individual health providers and institutions, and include payments, incentives and penalties. Financial interventions to providers include 'fee for service', where a provider is paid for the number and type of services delivered; capitation, where a provider is paid a set amount per patient for providing specific care; and provider salaried service, where a provider receives a basic salary for providing specific type of care to patients (EPOC 2002). Institution financial interventions include institution incentives, grants or penalties where the institution or group of providers receive direct or indirect financial penalties for inappropriate behaviour (EPOC 2002b). Organisational interventions include provider-orientated interventions (revision of professional roles, clinical multidisciplinary teams, formal integration of services, skills mix changes) and structural interventions (changes in settings/site of service delivery, changes in medical records systems, organisation of quality monitoring mechanisms). Regulatory interventions include any interventions that aim to change service delivery or costs by regulation or law, including changes in medical liability, management of patient complaints, peer review or licensure (EPOC 2002).

This review will assess professional, financial, organisational and regulatory interventions targeting health systems and providers, but will exclude interventions specifically targeting patients such as patient incentives or user fees.

\section{How the intervention might work}

For STIs, QI interventions aim to: increase levels of screening for asymptomatic infections to identify and treat those with the infection; provide early treatment of those with infection in order to break the chains of transmission; enhance contact tracing to prevent reinfection and onward transmission and ensure notification of diseases to support monitoring and surveillance (Low 2006). Other STI QI interventions seek to enhance the rescreening of clients who have previously presented with an STI, as studies have demonstrated that a past history of an STI is highly predictive of recurrent infection (Peterman 2006; Turner 2010). In the USA, it is recommended that all sexually-active females aged below 25 years should be routinely screened for chlamydia and gonorrhoea on an annual basis and that sexually-active young men in clinical settings with high prevalence of chlamydia should also be routinely screened, including in sexually transmitted disease (STD) clinics and correctional facilities (CDC 2015).

Screening for chlamydia and gonorrhoea is also recommended for older women who are at risk of infection, including those with a new partner, those with more than one sexual partner or those who are a contact of someone else with an STI. Universal screening for trichomoniasis is generally not recommended but advised only for women presenting with a vaginal discharge and those receiving care in high-prevalence settings such as STD clinics and correctional facilities (CDC 2015). The United States Preventive Services Task Force (USPSTF) does not recommend routine screening for syphilis among asymptomatic persons who are not at increased risk, due to the potential harms of screening such as costs, false positives and labelling/stigma (USPSTF 2014). Syphilis screening is only recommended for those at high risk and pregnant women at the first prenatal visit because of its high association with stillbirths, neonatal deaths and other complications (USPSTF 2009). Furthermore, Centers for Disease Control and Prevention (CDC) guidelines recommend that any person who tests positive for chlamydia or gonorrhoea and women who test 
positive for trichomoniasis should be rescreened at three months after treatment (CDC 2015). All of the aforementioned activities aim to reduce the transmission rates and overall burden of infection and associated complications. For instance, studies have found that screening for chlamydia in non-pregnant, high-risk females reduces the prevalence of chlamydia and the incidence of PID (Ostergaard 2000; Scholes 1996). Other studies have found that screening and treatment of chlamydia in pregnant women improves outcomes for both mother and child (Meyers 2007). Overall the aim of QI interventions targeting health services is to improve the clinical practice of the health providers in line with best practice guidelines, and effect organisational change in order to facilitate effective processes of care (Grol 2007). Interventions aim to facilitate changes that lead to better patient outcomes and system performance (Batalden 2007). However, how these interventions work will depend on the type of intervention, the context or external factors, the goal of the intervention, the target group/ population, and the characteristics of the health service and service providers (see Grol 2007 for detailed theoretical underpinnings of various QI interventions). For instance, professional interventions aim to: increase knowledge of best practice among providers by increasing access to educational materials and attendance at educational meetings and through educational outreach visits; influence practice through local opinion leaders who are 'educationally influential'; provide reminders to prompt health professionals; or provide a mechanism for audit and feedback (AF) (EPOC 2002b). Educational meetings impact on health professionals' competence and performance and ultimately on patient and population health outcomes by increasing health professionals' knowledge of best practice versus their current practice (Straus 2013). Pathman et al (Pathman 1996) describe a four-stage model - awareness, agreement, adoption and adherence; stages through which physicians progress in order for change to be adopted and sustained. As stated above, educational meetings may use a range of methods but it is generally accepted that more interactive workshops are more effective in changing clinical practice compared to didactic sessions (Straus 2013). Similarly, education materials aim to increase knowledge around best practice, however the effectiveness of these materials on health professionals' behaviour will depend on: the source of the material (its credibility and proximity to the health professionals); the channel (mode, frequency and duration); the message (clinical area, targeted behaviour, purpose and level of evidence); and the format (presentation, appearance and length) (Giguere 2012).

AF works through various mechanisms including enhancing practitioners' awareness of their current practice in relation to desired performance (Straus 2013), and changing norms and setting goals for change (Ivers 2012). However, as an intervention, AF is also influenced by other factors including quality of data provided, motivation of health providers, organisational support, other contextual variables and whether the feedback draws attention to the task or to the individual practitioner (Ivers 2012; Kluger 1996).
In addition, prerequisites for effective feedback include timeliness, individualisation, non-punitiveness and, to a lesser degree, customisation of the feedback (Hysong 2012).

Financial incentives act as an extrinsic source of motivation to effect behaviour change which may result in the desired change, but may inadvertently create a disincentive or other undesirable effects such as falsifying of reports, increase in quantity but not quality of care, or neglect of non-incentivised conditions/diseases (Straus 2013). In contrast, with organisational interventions, the focus is not on changing the individual's behavior, whether of the professional or patient, but on reforming the system and changing the structure of delivery of care, because inadequate performance is seen not as a failure of an individual, but as a failure of the system ( Grol 2007). Importantly, for all of the interventions discussed here, a range of factors interacting at the patient, health professional, organisational, economic and political levels will determine the effectiveness of interventions and a proper assessment of these factors must be made prior to implementation (Grol 2007).

\section{Why it is important to do this review}

Despite major advances and development of a range of innovative prevention and treatment strategies for the control of STIs, there is limited evidence about the impact of QI interventions on detection and management of curable STIs in primary care. Past Cochrane and non-Cochrane systematic reviews have determined the effectiveness of QI interventions on diabetic care (Renders 2009; Shojania 2006; Tricco 2012), hypertension (Walsh 2006) and musculoskeletal conditions (French 2010; Tzortziou 2008). Other reviews have assessed the effectiveness of single QI interventions on professional practice, for instance AF (Ivers 2012) and continuous quality improvement strategies (Brennan 2013). One Cochrane review assessed the effectiveness of a range of partner notification strategies for people with STIs (Ferreira 2013) and another has been proposed to assess interventions for screening for chlamydia (Low 2013); however both reviews do not specifically assess the effectiveness of QI interventions on detection and management of curable STIs within primary care.

A non-Cochrane systematic review assessed the efficacy of interventions targeting health services and patients in increasing screening for chlamydia, and found that significant increases in testing rates among females were associated with multifaceted interventions (relative risk (RR) 2.8 (95\% confidence interval (CI) 2.4 to 3.2) (Guy 2011); smaller increases in testing rates were associated with other interventions such as computer reminders (odds ratio (OR) $1.3,95 \%$ CI 1.1 to 1.4 ), linking chlamydia screening with Pap smears (OR 2.1, 95\% CI 1.3 to 3.4) and interactive educational workshops for clinical staff (RR 1.0, 95\% CI 0.8 to 1.2). Screening rates in males were significantly associated with a multifaceted quality improvement program that consisted of educational meetings for clinicians, audit and feedback and development of clinical teams (RR 3.0, 95\% CI 2.5 to 3.5) and a directive 
to all doctors to test all males aged 16 to 25 years (RR 7.7, 95\% CI 6.6 to 9.0). However this review was limited to chlamydia, to high-income countries and to English language publications. In addition, this review used a restricted definition of primary care, describing it as a health service that provides the first point of entry of care and as the ongoing focal point for all the patient's healthcare requirements. This definition potentially excludes other providers of primary care in low-income countries such as hospitals and other primary care service providers who may not be the ongoing focal point for all of the patient's healthcare requirements. Similarly another non-Cochrane review assessed the effectiveness of interventions in increasing rescreening for repeat chlamydia infection (Guy 2012). Mailed screening kits and reminders were most significantly associated with increased rescreening rates, however again this review was limited to chlamydia. In addition, both of these reviews were limited to screening which is only one aspect of sexual health service delivery.

This review therefore seeks to add to the existing knowledge about effective QI interventions designed to improve health providers' practice in the detection and management of the four most common curable STIs and also those designed to improve the broader health system's responsiveness to detection and management of these STIs. The review proposes to determine the effectiveness of STI QI interventions within primary care facilities and with primary care providers in order to resolve any conflicting evidence, explore the effectiveness of interventions in different settings and thus provide a basis for decision-making in these contexts.

\section{O B JECT IVES}

To assess the effectiveness and safety of quality improvement interventions for the detection and management of curable sexually transmitted infections in primary care.

\section{METHODS}

\section{Criteria for considering studies for this review}

\section{Types of studies}

We will include all published and unpublished randomised controlled trials (RCTs) including cluster-randomised trials and crossover trials. Since the focus of our review is quality improvement strategies, we will also include all published and unpublished non-randomised controlled trials (NRCTs), controlled before-after studies (CBAs), and interrupted time series (ITS) studies. We will exclude any ITS study that does not have a clearly-defined point in time when the intervention occurred, and does not have at least three data points before and three data points after the intervention (EPOC 2002b).

\section{Types of participants}

In this review, we will include interventions targeting primary care facilities and primary care providers. We use a broad definition of primary care that includes "all general health facilities which cover a broad range of presenting ailments which can be accessed by a wide range of patients on demand and not as a result of referral for specialist care" (Kaner 2007; Sanz-Cuesta 2012). We use this broad definition because in many low- and middle-income countries (LMICs), a significant proportion of patients access a range of health providers and facilities including private and public health outpatient/ambulatory facilities and pharmacies as their first point of entry to care (Marek 2005). The participants include:

1. Primary care facilities (including general practices, genitourinary medicine clinics, family planning clinics, community sexual health services, community pharmacies, and outpatient/ambulatory clinics); and

2. Primary care providers (including general practitioners, family practitioners, family physicians, nurses, community health workers, and community pharmacists).

We will include non-medical personnel including pharmacists and community health workers as long as they fit into the definition of primary care providers as defined above.

\section{Types of interventions}

We will include interventions that improve delivery of sexual health services for chlamydia, gonorrhoea, trichomoniasis and syphilis within primary care facilities and with primary care providers. We will classify the interventions implemented in the included studies as professional, organisational, financial and regulatory interventions in accordance with the Cochrane Effective Practice and Organisation of Care Group (EPOC) taxonomy of interventions (see EPOC 2002). Patient-oriented financial interventions including user fees, patient incentives, and co-payments, and patient-oriented organisational interventions such as mailorder pharmacies will be excluded from this review. Appendix 1 presents all of the categories of interventions that will be included in this review.

Consideration will be given to the intervention/s or combinations of interventions that were delivered, their intensity and frequency, who delivered the intervention, and the duration of the intervention. Comparisons will be made between the following:

- single intervention compared with standard practice or no intervention;

- single intervention compared with another single intervention;

- single intervention compared with multifaceted intervention (any combination, dose, frequency, duration, intensity); 
- multifaceted intervention (any combination, dose, frequency, duration, intensity) compared with standard practice or no intervention.

\section{Types of outcome measures}

The outcomes in the selected studies will depend on the local guidelines for each country/jurisdiction and also on the STI. Depending on the study design, comparisons of outcomes may be made between services or within the same service over time. For each type of infection, the following outcomes will be considered:

\section{Primary outcomes}

- Proportion of the target population screened/tested (effective screening rate).

- Average time to treatment.

- Proportion of cases rescreened/tested at three months.

- Proportion of the target population tested annually.

\section{Secondary outcomes}

- Proportion of diagnosed patients treated.

- Proportion of tests with a positive result (test positivity).

- Proportion of cases with confirmed contact tracing/partner notification.

- Proportion of cases re-infected.

- Proportion of cases notified to a central service.

- Proportion of false positives.

- Harm to patients, for example partner violence, abuse or suicide.

- Health professional outcomes: changes with regard to health professionals' behaviour, knowledge, attitudes or satisfaction.

- Prevalence and incidence of STIs.

- Incidence of pelvic inflammatory disease (women) and epididymitis (men) secondary to chlamydia.

- Health service outcome: costs.

- Adverse events

\section{Search methods for identification of studies}

We will develop a highly sensitive search strategy to identify as many relevant Randomized Controlled Trials (RCTs) and nonRCT designs as possible of quality improvement interventions for improving the detection and management of curable STIs in primary care, irrespective of their language, publication date and publication status (published, unpublished, in press, and in progress). We will use both electronic searching in bibliographic databases and handsearching, as described in the Cochrane Handbook for Systematic Reviews of Interventions (Higgins 2011).

The results of all searches will be downloaded and managed using Endnote bibliographic software. Duplicate records of the same study will be deleted.

\section{Electronic searches}

We will contact the information specialist of the Sexually Transmitted Infections Cochrane Review Group in order to implement a comprehensive search strategy to capture as many relevant RCTs and non RCTs as possible in electronic databases. For this purpose, we will use a combination of exploded controlled vocabulary (MeSH, Emtree, DeCS) and free-text terms (considering spelling variants, plurals, synonyms, acronyms and abbreviations), with field labels, truncation, proximity operators and boolean operators. The search strategy for MEDLINE, EMBASE and LILACS can be found in Appendix 2 (Electronic search strategies). We will modify the search strategy for other databases using appropriate syntax and vocabulary for those databases.

Specifically, we will search in the following electronic databases:

- MEDLINE, Ovid platform: inception to present.

- MEDLINE In-Process \& Other Non-Indexed Citations, Ovid platform: inception to present.

- MEDLINE Daily Update, Ovid platform: inception to present.

- EMBASE.com: inception to present.

- The Cochrane Central Register of Controlled Trials

(CENTRAL), Ovid platform: inception to present.

- LILACS, iAHx interface: inception to present.

- PsycINFO: inception to present.

- African Index Medicus (AIM), Virtual Health Library (VHL): inception to present.

- CINAHL (Cumulative Index to Nursing and Allied Health Literature): inception to present

We will use for MEDLINE, Cochrane highly sensitive search strategy for identifying RCTs: sensitivity and precision maximizing version (2008 revision), Ovid format (Higgins 2011), and an the Effective Practice and Organisation of Care (EPOC) methodology filter to identify non-RCT designs (Steed 2014).

These searches will be updated within six months before publication of the review.

\section{Searching other resources}

We will attempt to identify additional relevant RCTs and nonRCTs studies by using of the following methods:

1. Searching in the Sexually Transmitted Infections Cochrane Review Group's Specialized Register, which includes RCTs and controlled clinical trials, from 1944 to 2014, located through:

- Electronic searching in MEDLINE, EMBASE and CENTRAL.

- Online handsearching in those journals not indexed in MEDLINE or EMBASE, according to the journals' master list of the STI Cochrane Review Group.

2. Searching in trials registers:

Quality improvement interventions for improving the detection and management of curable sexually transmitted infections in primary 
- WHO International Clinical Trials Registry Platform ICTRP portal (http://apps.who.int/trialsearch/): inception to present.

- ClinicalTrials.gov (http://clinicaltrials.gov/): inception to present.

3. Searching in Web of Science: inception to present.

4. Searching for grey literature in System for Information on Grey Literature in Europe "OpenGrey" (http://www.opengrey.eu/): inception to present.

5. Searching by contacting with authors of all RCTs identified by others methods. A comprehensive list of RCTs included in the review along with the criteria for considering studies will be sent to the first author of each included study, asking for any additional studies published or unpublished that might be relevant.

6. Handsearching in journals that are not indexed in MEDLINE or EMBASE (according to the journals' master list of the Cochrane STI Review Group):

- Anatolian Journal of Obstetrics \& Gynecology;

- Current Medical Literature Gynecology \& Obstetrics;

- Current Obstetrics and Gynecology Reports;

- ISRN Obstetrics and Gynecology;

- Journal of South Asian Federation of Obstetrics \& Gynecology;

- Obstetrics and Gynecology International;

- Obstetrics Gynaecology and Reproductive Medicine;

- Sexual Science: the Newsletter of the Society for the Scientific Study of Sexuality and Sexualities.

7. Handsearching of conference proceeding abstracts in the following events:

- The International Society for Sexually Transmitted Diseases Research - ISSTDR (http://www.isstdr.org/): 2007, 2009, 2011, 2013 and 2015.

- The British Association for Sexual Health and HIV BASHH (http://www.bashh.org/): 2014 and 2015

- International Congress on Infectious Diseases - ICID ( http://www.isid.org/): 2010, 2012 and 2014.

- The International Union against Sexually Transmitted Infections - IUSTI (http://www.iusti.org/): 2011, 2012, 2013, 2014 and 2015.

- International Society for Infectious Diseases - ISID (http:// www.isid.org/): 2011, 2012, 2013, 2014 and 2015.

- International Meeting on Emerging Diseases and Surveillance - IMED (http://www.isid.org/): 2007, 2009, 2011, 2013 and 2014

- Interscience Conference on Antimicrobial Agents and Chemotherapy - ICAAC (http://www.icaac.org/): 2011, 2012, 2013, 2014 and 2015.

- The International Federation of Gynecology and Obstetrics - FIGO (http://www.figo2012.org/home/): 2009, 2012 and 2015.
8. Handsearching within previous systematic reviews and other relevant publications on the same topic.

9. Handsearching within reference lists of all relevant RCTs identified by others methods.

\section{Data collection and analysis}

\section{Selection of studies}

Using a form with predefined inclusion criteria, two review authors, Barbara Nattabi (BN) and Alice Rumbold (AR), will independently assess all the titles and abstracts of the studies for eligibility. We will assess and obtain full-text manuscripts for those studies that we agree are potentially eligible. For those articles for which we cannot agree, we will obtain the full text of the article to determine eligibility. If there are insufficient data in the reports to determine eligibility, we will contact the relevant authors for extra information. We will resolve all disagreements with discussion, and consultation with the third review author, Sajni Gudka (SG).

\section{Data extraction and management}

Two authors (BN, AR) will independently extract data using a specially designed standardised extraction form based on the Cochrane STI Group and EPOC Group data collection sheets. We will pilot the form with a representative number of studies and modify it if there are any missing data. The authors will extract data from every report associated with each eligible study. In instances where there is a single main report and a multitude of smaller reports of the same study, the data will be merged directly into a single data collection form. If there are multiple detailed manuscripts of the same study, data will be extracted from the most comprehensive report and extra data from the other reports will be extracted and included into a single data collection form. We will resolve any discrepancies through discussion or adjudication by the third author, SG.

Articles in languages other than English will be translated and data extracted in the process described above.

We will extract data on: the authors and citation; location of study and setting (low-, middle- and high-income countries); regional location of service (rural, urban); ethical approval; study design (randomised, non-randomised; CBA; ITS); inclusion and exclusion criteria; methods (duration, sequence generation, allocation sequence concealment, blinding); characteristics of study participants (primary care facilities and primary care providers); population risk profile (high, low or unclear risk of contracting an STI, as defined by authors); type of STI (chlamydia, gonorrhoea, trichomoniasis, syphilis); type and components of the intervention (professional, financial, organisational, regulatory); type and components of the comparison intervention; and outcomes (primary and secondary). 


\section{Assessment of risk of bias in included studies}

Two review authors will independently assess the risk of bias for each included RCT study using the criteria outlined in the Cochrane Handbook for Systematic Reviews of Interventions using the Cochrane Collaboration's 'Risk of bias' tool (Higgins 2011). We will use the ROBINS-I tool (Risk Of Bias In Non-randomized Studies - of Interventions) to assess the risk of bias in the non-randomised trials (Sterne 2014). Any disagreement will be resolved by discussion or by involving a third author.

The domains that we will assess for risk are:

(1) Random sequence generation (checking for possible selection bias)

We will describe for each included study the method used to generate the allocation sequence in sufficient detail to allow an assessment of whether it should produce comparable groups.

We will assess the methods as:

- low risk of bias (any truly random process, e.g. random number table; computer random number generator);

- high risk of bias (any non-random process, e.g. odd or even date of birth; hospital or clinic record number);

- unclear risk of bias.

(2) Allocation concealment (checking for possible selection bias)

We will describe for each included study the method used to conceal allocation to interventions prior to assignment and assess whether intervention allocation could have been foreseen in advance of, or during recruitment, or changed after assignment. We will assess the methods as:

- low risk of bias (e.g. telephone or central randomisation; consecutively-numbered sealed opaque envelopes);

- high risk of bias (open random allocation; unsealed or nonopaque envelopes, alternation; date of birth);

- unclear risk of bias.

(3.1) Blinding of participants and personnel (checking for possible performance bias)

We will describe for each included study the methods used, if any, to blind study participants and personnel from knowledge of which intervention a participant received. We will consider that studies were at low risk of bias if they were blinded, or if we judged that the lack of blinding was unlikely to affect results. We will assess blinding separately for different outcomes or classes of outcomes. We will assess the methods as:

- low, high or unclear risk of bias for participants;

- low, high or unclear risk of bias for personnel.

(3.2) Blinding of outcome assessment (checking for possible detection bias)

We will describe for each included study the methods used, if any, to blind outcome assessors from knowledge of which intervention a participant received. We will assess blinding separately for different outcomes or classes of outcomes.

We will assess methods used to blind outcome assessment as:
- low, high or unclear risk of bias.

(4) Incomplete outcome data (checking for possible attrition bias due to the amount, nature and handling of incomplete outcome data)

We will describe for each included study, and for each outcome or class of outcomes, the completeness of data including attrition and exclusions from the analysis. We will state whether attrition and exclusions were reported and the numbers included in the analysis at each stage (compared with the total randomised participants), reasons for attrition or exclusion where reported, and whether missing data were balanced across groups or were related to outcomes. Where sufficient information is reported, or can be supplied by the trial authors, we plan to re-include missing data in the analyses.

We will assess methods as:

- low risk of bias (e.g. no missing outcome data; missing outcome data balanced across groups);

- high risk of bias (e.g. numbers or reasons for missing data imbalanced across groups; 'as treated' analysis done with substantial departure of intervention received from that assigned at randomisation);

- unclear risk of bias.

\section{(5) Selective reporting (checking for reporting bias)}

We will describe for each included study how we investigated the possibility of selective outcome reporting bias and what we found. We will assess the methods as:

- low risk of bias (where it is clear that all of the study's prespecified outcomes and all expected outcomes of interest to the review have been reported);

- high risk of bias (where not all the study's pre-specified outcomes have been reported; one or more reported primary outcomes were not pre-specified; outcomes of interest are reported incompletely and so cannot be used; the study fails to include results of a key outcome that would have been expected to have been reported);

- unclear risk of bias.

(6) Other bias (checking for bias due to problems not covered by (1) to (5) above)

We will describe for each included study any important concerns we have about other possible sources of bias.

(7) Overall risk of bias

We will make explicit judgements about whether studies were at high risk of bias, according to the criteria given in the Cochrane Handbook (Higgins 2011). With reference to (1) to (6) above, we plan to assess the likely magnitude and direction of the bias and whether we consider it is likely to impact on the findings. We plan to explore the impact of the level of bias through undertaking sensitivity analyses - see Sensitivity analysis.

Risk of bias in cross-over trials

In addition we shall assess for risk in cross-over trials, specifically:

- if use of a cross-over trial was appropriate for the study;

Quality improvement interventions for improving the detection and management of curable sexually transmitted infections in primary 
- if the order of receiving the intervention was randomised;

- if the trial was not biased from carry-over effects; and

- if unbiased data are available (Higgins 2011).

Risk of bias in controlled before-after and interrupted time series studies

In addition to using the ROBINS-I tool to assess the risk of bias in CBA and ITS studies, ITS studies will be assessed using seven extra criteria:

- if the intervention was independent of other changes;

- if the shape of the intervention effect was pre-specified;

- if the intervention was unlikely to affect data collection;

- if knowledge of the allocated interventions was adequately prevented during the study;

- if incomplete outcome data were adequately addressed;

- if the study was free from selective outcome reporting;

- if the study was free from other risks of bias (EPOC 2008).

\section{Measures of treatment effect}

For dichotomous data, the results will be presented as a summary risk ratio (RR) with $95 \%$ confidence intervals (CIs). For studies that report continuous outcomes that are measured in the same way, we will use the mean difference. For studies that use different methods to assess the same outcome, we will use the standardised mean difference. For time-to-event outcomes, we will present treatment effects as overall hazard ratios with $95 \%$ CIs.

\section{Unit of analysis issues}

\section{Randomised trials}

We plan to include cluster-randomised trials as well as individually-randomised trials. For cluster-randomised trials, we will adjust the sample size using the methods described in the Cochrane Handbook (Higgins 2011) using an estimate of the intracluster correlation co-efficient (ICC) derived from the trial (if possible), from a similar trial or from a study of a similar population. If we use ICCs from other sources, we will report this and conduct sensitivity analyses to investigate the effect of variation in the ICC. If we identify both cluster-randomised trials and individually-randomised trials, we plan to synthesise the relevant information. We will consider it reasonable to combine the results from both if there is little heterogeneity between the study designs, and the interaction between the effect of the intervention and the choice of randomisation unit is considered to be unlikely.

\section{Multi-arm studies}

If we identify eligible multi-arm studies (more than one treatment group), we will combine treatment groups if appropriate, and create a single pair-wise comparison. We will not double count partic- ipants, according to the methods described in the Cochrane Handbook (Higgins 2011).

\section{Dealing with missing data}

If data related to the methods are missing, or if the methods are not clear, we will contact the original investigators for clarification. If outcome data are missing, we will contact the original investigators to request the missing data. If we fail to obtain missing outcome data, we will conduct an analysis on the outcome variables on an intention-to-treat basis, i.e. we aim to include all participants as they were randomised, regardless of what occurred subsequently. Where summary data are missing, we will calculate or estimate the missing value from available statistics in the paper. For instance we will calculate the standard deviation from standard errors, confidence intervals, $t$ values and $\mathrm{P}$ values. If these statistics are not available, we will impute the missing values e.g. we will use standard deviations from one or more other studies in the meta-analysis or from other studies in another meta-analysis (Higgins 2011). However, imputation of standard deviations will not be conducted if a majority of studies have missing standard deviations. We will report the assumptions we have made in our results tables.

\section{Assessment of heterogeneity}

We will make an assessment of whether to conduct a meta-analysis by assessing for heterogeneity between the included studies. We will conduct a visual inspection of the forest plots and use the Chi ${ }^{2}$ test and $\mathrm{I}^{2}$ statistic to measure the level of heterogeneity. We will consider that heterogeneity is substantial, and hence meta-analysis impossible, if the CIs of the effect estimates of the individual studies have significantly poor overlap, if $\mathrm{I}^{2}$ is greater than $40 \%$ or if the $\mathrm{P}$ value is less than 0.10 in the $\mathrm{Chi}^{2}$ test for heterogeneity.

\section{Assessment of reporting biases}

If there are 10 or more studies in the meta-analysis we will investigate reporting biases (such as publication bias) using funnel plots. We will assess funnel plot asymmetry visually, as well as using formal tests. For continuous outcomes we will use the test proposed by Egger 1997, and for dichotomous outcomes we will use the test proposed by Harbord 2006. If we detect asymmetry in any of these tests or it is suggested by a visual assessment, we will perform exploratory analyses to investigate it.

\section{Data synthesis}

We will use narrative synthesis to describe the results of trials where there are too few studies for meta-analysis or where we consider that meta-analysis is not clinically meaningful. We will use forest plots to display results of trials examining the same outcome. If we can reasonably assume that the studies are estimating the 
same intervention and the studies' population and methods are judged sufficiently similar, we will use a fixed-effect meta-analysis for combining data. However we expect to find considerable heterogeneity due to diversity in countries, health systems, participants (primary healthcare services and providers), interventions being evaluated and study designs. Furthermore we expect that many eligible studies such as cluster-randomised studies will have 'unit of analysis' errors. If we detect considerable heterogeneity, or there are too few studies, we will not perform meta-analysis to combine the data.

Should we have a sufficient number of comparable studies, i.e. based on population, study design, intervention and comparisons, we will separate our analysis by RCTs, NRCTs, CBAs and ITS. For RCTs, we will use a random-effects model to estimate the average effect across trials as we expect that the studies will be naturally heterogeneous. The results will be presented as the summary RR (95\% CI) with $\mathrm{I}^{2}$ and tau ${ }^{2}$ estimates for outcomes reporting dichotomous data. We will calculate the mean difference for outcomes reporting continuous data. If it is possible to conduct a meta-analysis with at least three studies using a random-effects model, we will also calculate a prediction interval. A prediction interval is a CI which takes into account heterogeneity and provides a wider predicted range for the true treatment effect of an intervention in an individual setting, making it of more relevance to clinical practice and decision making (Riley 2011).

We will initially include all trials and conduct a sensitivity analysis, excluding any non-randomised studies. If the non-randomised studies are deemed relatively homogenous with a low risk of bias, we will similarly combine data using meta-analysis. For the metaanalysis of non-randomised studies we will use the estimated intervention effect and its standard error (or CI) and data will be pooled using the generic inverse-variance method (Higgins 2011). All statistical analysis will be carried out using the Review Manager software (RevMan 2014).

\section{Subgroup analysis and investigation of heterogeneity}

For data that can be included in a meta-analysis, we plan to investigate any identified heterogeneity using subgroup analyses and sensitivity analyses. If substantial heterogeneity is detected, we will first consider whether it is meaningful to calculate an overall summary effect measure, and if so, we will use a random-effects analysis to do so.
We will carry out the following subgroup analyses for primary outcomes:

1. location of study (low-, middle- and high-income countries)

2. regional location of service (rural, urban)

3. characteristics of study participants (primary care facilities and primary care providers);

4. population risk profile (high, low or unclear risk of contracting an STI as defined by the study authors);

5. type and components of the intervention (professional, financial, organisational, regulatory).

Where data can be included in a meta-analysis, we will assess subgroup differences by interaction tests available within RevMan (RevMan 2014). We will report the results of subgroup analyses quoting the $\mathrm{Chi}^{2}$ statistic and $\mathrm{P}$ value, and the interaction test $\mathrm{I}^{2}$ value.

\section{Sensitivity analysis}

We will carry out sensitivity analyses to explore the effect of study quality assessed by concealment of allocation (where applicable), high attrition rates, or both. Studies with high risk of bias will be excluded from the meta-analysis in order to assess whether this made any difference to the overall result. Where cluster-randomised studies are included we will also perform a sensitivity analysis based on the unit of randomisation. The sensitivity analyses will also compare the results of the meta-analysis of randomised trials with the meta-analysis of non-randomised trials, to determine the influence of difference study designs on the overall effect estimate.

For studies that could not be included in a meta-analysis, we will descriptively compare the results of studies with high risk of bias and studies with low risk of bias.

\section{ACKNOWLEDGEMENTS}

The authors thank the Cochrane Sexually Transmitted Infections editorial team for their support, advice and assistance throughout the preparation of this protocol. Barbara Nattabi is supported by a NHMRC Early Career Research Fellowship and Alice Rumbold is supported by a NHMRC Career Development Fellowship. The Western Australian Centre for Rural Health receives funding from the Australian Federal Department of Health. 


\section{R E F E R E N C E S}

\section{Additional references}

\section{ASHA 2014}

Australasian Sexual Health Alliance. Australian STI management guidelines for use in primary care. sti.guidelines.org.au/ (accessed 8 April 2016).

Batalden 2007

Batalden PB, Davidoff F. What is "quality improvement" and how can it transform healthcare?. Quality \& Safety in Health Care 2007;16(1):2-3.

\section{Belkacem 2013}

Belkacem A, Caumes E, Ouanich J, Jarlier V, Dellion S, Cazenave B, et al. Changing patterns of disseminated gonococcal infection in France: cross-sectional data 20092011. Sexually Transmitted Infections 2013;89(8):613-5.

\section{Bowden 2000}

Bowden FJ, Garnett GP. Trichomonas vaginalis epidemiology: parameterising and analysing a model of treatment interventions. Sexually Transmitted Infections 2000;76(4):248-56.

Brehaut 2012

Brehaut JC, Eva KW. Building theories of knowledge translation interventions: use the entire menu of constructs. Implementation Science 2012;7:114.

Brennan 2013

Brennan S, McKenzie JE, Whitty P, Buchan H, Green S. Continuous quality improvement: effects on professional practice and healthcare outcomes. Cochrane Database of Systematic Reviews 2013, Issue 10. [DOI: 10.1002/ 14651858.CD003319.pub2]

\section{CDC 2014}

Centers for Disease Control and Prevention. Sexually Transmitted Disease Surveillance 2013. Atlanta: U.S. Department of Health and Human Services 2014.

CDC 2015

Centers for Disease Control and Prevention. Sexually transmitted diseases treatment guidelines, 2015. Morbidity and Mortality Weekly Report 2015;64(RR3):1-137.

\section{Chandeying 2000}

Chandeying V, Skov S, Duramad P, Makepeace B, Ward M, Khunigij P. The prevalence of urethral infections amongst asymptomatic young men in Hat Yai, southern Thailand. International Journal of STD \& AIDS 2000;11(6):402-5.

Coleman 2013

Coleman JS, Gaydos CA, Witter F. Trichomonas vaginalis vaginitis in obstetrics and gynecology practice: new concepts and controversies. Obstetrical \& Gynecological Survey 2013;68(1):43-50.

Colquhoun 2013

Colquhoun HL, Brehaut JC, Sales A, Ivers N, Grimshaw $\mathrm{J}$, Michie S, et al. A systematic review of the use of theory in randomized controlled trials of audit and feedback. Implementation Science 2013;8:66.
Cotch 1997

Cotch MF, Pastorek JG, Nugent RP, Hillier SL, Gibbs RS, Martin $\mathrm{DH}$, et al. Trichomonas vaginalis associated with low birth weight and preterm delivery. Sexually Transmitted Diseases 1997;24(6):353-60.

Donovan 2004

Donovan B. Sexually transmissible infections other than HIV. Lancet 2004;363(9408):545-56.

\section{ECDC 2015}

European Centre for Disease Prevention and Control. Sexually transmitted infections in Europe 2013. Stockholm: ECDC 2015.

\section{Egger 1997}

Egger M, Davey Smith G, Schneider M, Minder C. Bias in meta-analysis detected by a simple, graphical test. $B M J$ 1997;315(7109):629-34.

\section{EPOC 2002}

Effective Practice, Organisation of Care (EPOC). EPOC taxonomy 2002. epoc-old.cochrane.org/epoc-taxonomy. Oslo: Norwegian Knowledge Centre for the Health Services, (accessed 21 November 2015).

\section{EPOC 2002b}

Cochrane Effective Practice and Organisation of Care Review Group (EPOC). Data collection checklist. http:/ /epoc.cochrane.org/sites/epoc.cochrane.org/files/uploads/ datacollectionchecklist.pdf.. Ottawa, Canada, (accessed 11 November 2015).

\section{EPOC 2008}

Cochrane Effective Practice and Organisation of Care Review Group (EPOC). Suggested risk of bias criteria for EPOC reviews. epoc.cochrane.org/ sites/epoc.cochrane.org/files/uploads/Suggested $\% 20$ risk $\% 20$ of $\% 20$ bias $\% 20$ criteria $\% 20$ for $\% 20$ EPOC $\% 20$ reviews.pdf (accessed 6 August 2016).

\section{Ferreira 2013}

Ferreira A, Young T, Mathews C, Zunza M, Low N. Strategies for partner notification for sexually transmitted infections, including HIV. Cochrane Database of Systematic Reviews 2013, Issue 10. [DOI: 10.1002/ 14651858.CD002843.pub2]

\section{Fleming 1999}

Fleming DT, Wasserheit JN. From epidemiological synergy to public health policy and practice: the contribution of other sexually transmitted diseases to sexual transmission of HIV infection. Sexually Transmitted Infections 1999;75(1): 3-17.

\section{Flodgren 2011}

Flodgren G, Parmelli E, Doumit G, Gattellari M, O'Brien MA, Grimshaw J, et al. Local opinion leaders: effects on professional practice and health care outcomes. Cochrane Database of Systematic Reviews 2011, Issue 8. [DOI: 10.1002/14651858.CD000125.pub4] 


\section{Forsetlund 2009}

Forsetlund L, Bjorndal A, Rashidian A, Jamtvedt G, O'Brien $\mathrm{MA}$, Wolf $\mathrm{F}$, et al. Continuing education meetings and workshops: effects on professional practice and health care outcomes. Cochrane Database of Systematic Reviews 2009, Issue 2. [DOI: 10.1002/14651858.CD003030.pub2]

French 2010

French SD, Green S, Buchbinder R, Barnes H. Interventions for improving the appropriate use of imaging in people with musculoskeletal conditions. Cochrane Database of Systematic Reviews 2010, Issue 1. [DOI: 10.1002/ 14651858.CD006094.pub2]

Garrow 2002

Garrow SC, Smith DW, Harnett GB. The diagnosis of chlamydia, gonorrhoea, and trichomonas infections by self obtained low vaginal swabs, in remote northern Australian clinical practice. Sexually Transmitted Infections 2002;78(4): $278-81$.

Genc 2000

Genc M, Ledger WJ. Syphilis in pregnancy. Sexually Transmitted Infections 2000;76(2):73-9.

\section{Giguere 2012}

Giguère A, Légaré F, Grimshaw J, Turcotte S, Fiander M, Grudniewicz A, et al. Printed educational materials: effects on professional practice and healthcare outcomes. Cochrane Database of Systematic Reviews 2012, Issue 10. [DOI: 10.1002/14651858.CD004398.pub3]

Grol 2007

Grol RPTM, Bosch MC, Hulscher MEJL, Eccles MP, Wensing M. Planning and studying improvement in patient care: the use of theoretical perspectives. Milbank Quarterly 2007;85(1):93-138

Guy 2011

Guy RJ, Ali H, Liu B, Poznanski S, Ward J, Donovan B, et al. Efficacy of interventions to increase the uptake of chlamydia screening in primary care: a systematic review. BMC Infectious Diseases 2011;11(1):211.

\section{Guy 2012}

Guy R, Hocking J, Low N, Ali H, Bauer HM, Walker $\mathrm{J}$, et al. Interventions to increase rescreening for repeat chlamydial infection. Sexually Transmitted Diseases 2012;39 (2):136-46.

\section{Harbord 2006}

Harbord RM, Egger M, Sterne JA. A modified test for small-study effects in meta-analyses of controlled trials with binary endpoints. Statistics in Medicine 2006;25(20): 3443-57.

Higgins 2011

Higgins JPT, Green S (editors). Cochrane Handbook for Systematic Reviews of Interventions Version 5.1.0 (updated March 2011). The Cochrane Collaboration, 2011. Available from www.cochrane-handbook.org.

\section{Hysong 2012}

Hysong SJ, Teal CR, Khan MJ, Haidet P. Improving quality of care through improved audit and feedback. Implementation Science 2012;7:45.
Ivers 2012

Ivers N, Jamtvedt G, Flottorp S, Young JM, OdgaardJensen J, French SD, et al. Audit and feedback: effects on professional practice and healthcare outcomes. Cochrane Database of Systematic Reviews 2012, Issue 6. [DOI: 10.1002/14651858.CD000259.pub3]

\section{Johnston 2008}

Johnston VJ, Mabey DC. Global epidemiology and control of Trichomonas vaginalis. Current Opinion in Infectious Diseases 2008;21(1):56-64.

Kaner 2007

Kaner EFS, Dickinson HO, Beyer FR, Campbell F, Schlesinger C, Heather N, et al. Effectiveness of brief alcohol interventions in primary care populations. Cochrane Database of Systematic Reviews 2007, Issue 2. [DOI: 10.1002/14651858.CD004148.pub3]

Kirby 2015

The Kirby Institute. HIV, viral hepatitis and sexually transmissible infections in Australia Annual Surveillance Report 2015. Sydney, NSW: The Kirby Institute 2015.

\section{Kluger 1996}

Kluger AN, DeNisi A. The effects of feedback interventions on performance: a historical review, a meta-analysis, and a preliminary feedback intervention theory. Psychological Bulletin 1996;119(2):254-84.

Low 2006

Low N, Broutet N, Adu-Sarkodie Y, Barton P, Hossain M, Hawkes S. Global control of sexually transmitted infections. Lancet 2006;368(9551):2001-16.

\section{Low 2013}

Low N, Redmond S, Uusküla A, van Bergen J, Ward H, Andersen B, et al. Screening for genital chlamydia infection. Cochrane Database of Systematic Reviews 2013, Issue 12. [DOI: 10.1002/14651858.CD010866]

\section{Marek 2005}

Marek T, O'Farrell C, Yamamoto C, Zable I. Trends and opportunities in public-private partnerships to improve health service delivery in Africa. Africa Region Human Development Working Paper Series. Washington (DC): World Bank 2005.

\section{Meyers 2007}

Meyers DS, Halvorson H, Luckhaupt S, U.S. Preventive Services Task Force. Screening for chlamydial infection: an evidence update for the U.S. Preventive Services Task Force. Annals of Internal Medicine 2007;147(2):135-42.

Mitchell 2014

Mitchell H, Lewis D, Marsh K, Hughes G. Distribution and risk factors of Trichomonas vaginalis infection in England: an epidemiological study using electronic health records from sexually transmitted infection clinics, 20092011. Epidemiology and Infection 2014;142(8):1678-87.

Nasser 2007

Nasser M, Oxman AD, Paulsen E, Fedorowicz Z. Local consensus processes: effects on professional practice and health care outcomes. Cochrane Database 
of Systematic Reviews 2007, Issue 1. [DOI: 10.1002/ 14651858.CD003165.pub3]

\section{Newman 2015}

Newman L, Rowley J, Vander Hoorn S, Wijesooriya NS, Unemo M, Low N, et al. Global estimates of the prevalence and incidence of four curable sexually transmitted infections in 2012 based on systematic review and global reporting. PLos One 2015;10(12):e0143304.

Ng 2005

$\mathrm{Ng}$ LK, Martin IE. The laboratory diagnosis of Neisseria gonorrhoeae. Canadian Journal of Infectious Diseases \& Medical Microbiology 2005;16(1):15-25.

OBrien 2007

O'Brien MA, Rogers S, Jamtvedt G, Oxman AD, OdgaardJensen J, Kristoffersen DT, et al. Educational outreach visits: effects on professional practice and health care outcomes. Cochrane Database of Systematic Reviews 2007, Issue 4. [DOI: 10.1002/14651858.CD000409.pub2]

Ostergaard 2000

Ostergaard L, Andersen B, Moller JK, Olesen F. Home sampling versus conventional swab sampling for screening of Chlamydia trachomatis in women: a cluster-randomized 1-year follow-up study. Clinical Infectious Diseases 2000;31 (4):951-7.

\section{PAHO 2015}

Pan American Health Organization. Guidance on syphilis testing in Latin America and the Caribbean: improving uptake, interpretation, and quality of testing in different clinical settings. Washington (DC): Pan American Health Organization 2015.

\section{Panaretto 2006}

Panaretto KS, Lee HM, Mitchell MR, Larkins SL, Manessis V, Buettner PG, et al. Prevalence of sexually transmitted infections in pregnant urban Aboriginal and Torres Strait Islander women in northern Australia. Australian and New Zealand Journal of Obstetrics and Gynaecology 2006;46(3): 217-24.

\section{Pathman 1996}

Pathman DE, Konrad TR, Freed GL, Freeman VA, Koch GG. The awareness-to-adherence model of the steps to clinical guideline compliance: the case of pediatric vaccine recommendations. Medical Care 1996;34(9):873-89.

\section{Peeling 1996}

Peeling RW, Brunham RC. Chlamydiae as pathogens: new species and new issues. Emerging Infectious Diseases 1996;2 (4):307-19.

Peipert 2003

Peipert J. Genital chlamydial infections. New England Journal of Medicine 2003;349(25):2424-30.

\section{Peterman 2006}

Peterman TA, Tian LH, Metcalf CA, Satterwhite CL, Malotte CK, DeAugustine N, et al. High incidence of new sexually transmitted infections in the year following a sexually transmitted infection: a case for rescreening. Annals of Internal Medicine 2006;145(8):564-72.
Plsek 1999

Plsek PE. Quality improvement methods in clinical medicine. Pediatrics 1999;103(1 Suppl E):203-14.

\section{Poole 2013}

Poole DN, McClelland RS. Global epidemiology of Trichomonas vaginalis. Sexually Transmitted Infections 2013; 89(6):418-22.

\section{Renders 2009}

Renders CM, Valk GD, Griffin SJ, Wagner E, van Eijk JThM, Assendelft WJJ. Interventions to improve the management of diabetes mellitus in primary care, outpatient and community settings. Cochrane Database of Systematic Reviews 2009, Issue 1. [DOI: 10.1002/ 14651858.CD001481]

RevMan 2014 [Computer program] Nordic Cochrane Centre, The Cochrane Collaboration. Review Manager (RevMan). Copenhagen: The Nordic Cochrane Centre, The Cochrane Collaboration. Version 5.3. Copenhagen (DK): Nordic Cochrane Centre, The Cochrane Collaboration, 2014.

\section{Riley 2011}

Riley RD, Higgins JP, Deeks JJ. Interpretation of random effects meta-analyses. BMJ 2011;342:d549.

\section{Sanz-Cuesta 2012}

Sanz-Cuesta T, López-Alcalde J, Del Cura-González I, Escortell-Mayor E, Martín-Fernández J, GómezGascón T, et al. Professional interventions to implement guidelines to prevent hazardous alcohol consumption by patients in primary care settings. Cochrane Database of Systematic Reviews 2012, Issue 7. [DOI: 10.1002/ 14651858.CD004630.pub2]

\section{Satterwhite 2013}

Satterwhite CL, Torrone E, Meites E, Dunne EF, Mahajan $\mathrm{R}$, Ocfemia MC, et al. Sexually transmitted infections among US women and men: prevalence and incidence estimates, 2008. Sexually Transmitted Diseases 2013;40(3): 187-93.

Scholes 1996

Scholes D, Stergachis A, Heidrich FE, Andrilla H, Holmes KK, Stamm WE. Prevention of pelvic inflammatory disease by screening for cervical chlamydial infection. New England Journal of Medicine 1996;334(21):1362-6.

Shojania 2004

Shojania KG, McDonald KM, Wachter RM, Owens DK. Closing the quality gap: a critical analysis of quality improvement strategies, Volume 1-Series Overview and Methodology. Technical Review 9 (Contract No. 290-020017 to the Stanford University-UCSF Evidence-based Practices Center). AHRQ Publication No. 04-0051-1. Rockville (MD): Agency for Healthcare Research and Quality (US) 2004.

\section{Shojania 2006}

Shojania KG, Ranji SR, McDonald KM, Grimshaw JM, Sundaram V, Rushakoff RJ, et al. Effects of quality improvement strategies for type 2 diabetes on glycemic 
control: a meta-regression analysis. Journal of the American Medical Association 2006;296(4):427-40.

\section{Shojania 2009}

Shojania KG, Jennings A, Mayhew A, Ramsay CR, Eccles MP, Grimshaw J. The effects of on-screen, point of care computer reminders on processes and outcomes of care. Cochrane Database of Systematic Reviews 2009, Issue 3. [DOI: 10.1002/14651858.CD001096.pub2]

\section{Steed 2014}

Steed L, Kassavou A, Madurasinghe VW, Edwards EA, Todd A, Summerbell CD, et al. Community pharmacy interventions for health promotion: effects on professional practice and health outcomes. Cochrane Database of Systematic Reviews 2014, Issue 7. [DOI: 10.1002/ 14651858.CD011207]

\section{Sterne 2014}

Sterne JAC, Higgins JPT, Reeves BC, on behalf of the development group for ROBINS-I. A tool for assessing risk of bias: in non-randomized studies of interventions, Version 7: March 2016. www.riskofbias.info (accessed 21 July 2016).

\section{Straus 2013}

Straus SE, Tetroe J, Graham ID. Knowledge Translation in Health Care: Moving from Evidence to Practice. 2nd Edition. West Sussex, UK: Wiley Blackwell, 2013.

Swygard 2004

Swygard H, Sena AC, Hobbs MM, Cohen MS. Trichomoniasis: clinical manifestations, diagnosis and management. Sexually Transmitted Infections 2004;80(2): $91-5$.

Tricco 2012

Tricco AC, Ivers NM, Grimshaw JM, Moher D, Turner L, Galipeau J, et al. Effectiveness of quality improvement strategies on the management of diabetes: a systematic review and meta-analysis. Lancet 2012;379(9833): 2252-61.

Turner 2002

Turner CF, Rogers SM, Miller HG, Miller WC, Gribble JN, Chromy JR, et al. Untreated gonococcal and chlamydial infection in a probability sample of adults. Journal of the American Medical Association 2002;287(6):726-33.

Turner 2010

Turner AN, Feldblum PJ, Hoke TH. Baseline infection with a sexually transmitted disease is highly predictive of reinfection during follow-up in Malagasy sex workers.

Sexually Transmitted Diseases 2010;37(9):559-62.

\section{Tzortziou 2008}

Tzortziou V, Morrissey D, Underwood M. Professional interventions for general practitioners on the management of musculoskeletal conditions. Cochrane Database of Systematic Reviews 2008, Issue 4. [DOI: 10.1002/ 14651858.CD007495]

Uddin 2011

Uddin RNN, Ryder N, McNulty AM, Wray L, Donovan B. Trichomonas vaginalis infection among women in a low prevalence setting. Sexual Health 2011;8(1):65-8.

\section{USPSTF 2009}

U.S. Preventive Services Task Force. Screening for syphilis infection in pregnancy: U.S. Preventive Services Task Force Reafrmation Recommendation Statement. Annals of Internal Medicine 2009;150:705-9.

\section{USPSTF 2014}

U.S. Preventive Services Task Force. Screening for syphilis infection: Recommendation Statement 2014. www.uspreventiveservicestaskforce.org/Page/Document/ UpdateSummaryFinal/syphilis-infection-screening?ds=1\& $s=$ syphilis. (accessed 20 October 2015).

\section{Walsh 2006}

Walsh JM, McDonald KM, Shojania KG, Sundaram V, Nayak S, Lewis R, et al. Quality improvement strategies for hypertension management: a systematic review. Medical Care 2006;44(7):646-57.

\section{Ward 2010}

Ward H, Ronn MM. Contribution of sexually transmitted infections to the sexual transmission of HIV. Current Opinion in HIV and AIDS 2010;5(4):305-10.

\section{WHO 2012}

World Health Organization. Global incidence and prevalence of selected curable sexually transmitted infections - 2008. Geneva: World Health Organization, Department of Reproductive Health and Research 2012.

\section{WHO 2015}

World Health Organization. Sexually Transmitted Infections (STIs) Fact sheet No. 110. www.who.int/ mediacentre/factsheets/fs110/en/ (accessed 20 July 2016).

* Indicates the major publication for the study 


\section{A P P E N D I C E S}

\section{Appendix I. Types of interventions from EPOC taxonomy}

The review will assess all quality improvement (QI) interventions listed in the EPOC taxonomy (EPOC 2002b), including:

1. Professional interventions

a. Distribution of educational materials: distribution of published or printed recommendations for clinical care, including clinical practice guidelines, audio-visual materials and electronic publications. The materials may have been delivered personally or through mass mailings.

b. Educational meetings: healthcare providers who have participated in conferences, lectures, workshops or traineeships.

c. Local consensus processes : inclusion of participating providers in discussion to ensure that they agreed that the chosen clinical problem was important and the approach to managing the problem was appropriate.

d. Educational outreach visits: use of a trained person who met with providers in their practice settings to give information with the intent of changing the provider's practice. The information given may have included feedback on the performance of the provider(s). e. Local opinion leaders: use of providers nominated by their colleagues as 'educationally influential'. The investigators must have explicitly stated that their colleagues identified the opinion leaders.

f. Patient-mediated interventions: new clinical information (not previously available) collected directly from patients and given to the provider, e.g. depression scores from an instrument.

g. Audit and feedback : any summary of clinical performance of health care over a specified period of time. The summary may also have included recommendations for clinical action. The information may have been obtained from medical records, computerised databases, or observations from patients.

The following interventions are excluded:

- Provision of new clinical information not directly reflecting provider performance which was collected from patients, e.g. scores on a depression instrument, abnormal test results. These interventions should be described as patient mediated.

- Feedback of individual patients' health record information in an alternate format (e.g. computerised). These interventions should be described as organisational.

h. Reminders: Patient- or encounter-specific information, provided verbally, on paper or on a computer screen, which is designed or intended to prompt a health professional to recall information. This would usually be encountered through their general education; in the medical records or through interactions with peers, and so remind them to perform or avoid some action to aid individual patient care. Computer-aided decision support and drugs dosage are included.

i. Marketing: Use of personal interviewing, group discussion ('focus groups'), or a survey of targeted providers to identify barriers to change and subsequent design of an intervention that addresses identified barriers.

j. Mass media: (i) varied use of communication that reached great numbers of people including television, radio, newspapers, posters, leaflets, and booklets, alone or in conjunction with other interventions; (ii) targeted at the population level.

k. Other

2. Financial interventions

a. Provider interventions

i. Fee-for-service : provider has been paid for number and type of service delivered.

ii. Prepaid: no other description.

iii. Capitation: provider was paid a set amount per patient for providing specific care.

iv. Provider salaried service: provider received basic salary for providing specific care.

v. Prospective payment : provider was paid a fixed amount for health care in advance.

vi. Provider incentives: provider received direct or indirect financial reward or benefit for doing specific action.

vii. Institution incentives: institution or group of providers received direct or indirect financial rewards or benefits for doing specific action.

viii. Provider grant/allowance: provider received direct or indirect financial reward or benefit not tied to specific action.

ix. Institution grant/allowance: institution or group of providers received direct or indirect financial reward or benefit not tied to specific action.

Quality improvement interventions for improving the detection and management of curable sexually transmitted infections in primary 
x. Provider penalty : provider received direct or indirect financial penalty for inappropriate behaviour.

xi. Institution penalty: institution or group of providers received direct or indirect financial penalty for inappropriate behaviour.

xii. Formulary: added or removed from reimbursable available products.

xiii. Other.

\section{Organisational interventions}

a. Provider-orientated interventions

i. Revision of professional roles: also known as 'professional substitution', 'boundary encroachment' and includes the shifting of roles among health professionals. For example, nurse midwives providing obstetrical care; pharmacists providing drug counselling that was formerly provided by nurses and physicians; nutritionists providing nursing care; physical therapists providing nursing care. Also includes expansion of role to include new tasks.

ii. Clinical multidisciplinary teams : creation of a new team of health professionals of different disciplines or additions of new members to the team who work together to care for patients.

iii. Formal integration of services: bringing together of services across sectors or teams or the organisation of services to bring all services together at one time also sometimes called 'seamless care'.

iv. Skill mix changes : changes in numbers, types or qualifications of staff.

v. Continuity of care: including one or many episodes of care for inpatients or outpatients.

- Arrangements for follow-up.

- Case management (including co-ordination of assessment, treatment and arrangement for referrals).

vi. Satisfaction of providers with the conditions of work and the material and psychic rewards, e.g. interventions to 'boost morale'. vii. Communication and case discussion between distant health professionals, e.g. telephone links; telemedicine; there is a television/ video link between specialist and remote nurse practitioners.

viii. Other .

b. Structural interventions

i. Changes in the setting/site of service delivery, e.g. moving a family planning service from a hospital to a school.

ii. Changes in physical structure, facilities and equipment, e.g. change of location of nursing stations, inclusion of equipment where technology in question is used in a wide range of problems and is not disease specific, for example an MRI scanner.

iii. Changes in medical records systems, e.g. changing from paper to computerised records, patient tracking systems.

iv. Changes in scope and nature of benefits and services.

v. Presence and organisation of quality monitoring mechanisms.

vi. Ownership, accreditation, and affiliation status of hospitals and other facilities.

vii. Staff organisation .

viii. Other.

4. Regulatory interventions .

Any intervention that aims to change health services delivery or costs by regulation or law. (These interventions may overlap with organisational and financial interventions).

a. Changes in medical liability.

b. Management of patient complaints .

c. Peer review .

d. Licensure.

e. Other .

Quality improvement interventions for improving the detection and management of curable sexually transmitted infections in primary 


\section{Appendix 2. Search strategy:}

\section{MEDLINE and CENTRAL (Ovid platform)}

1 exp Chlamydia trachomatis/

2 Chlamydia.ti,ab.

3 chlamidia.ti,ab.

4 chlamidiasis.ti,ab.

5 chlamydiasis.ti,ab.

6 chlamidiosis.ti,ab.

7 (urogenital adj5 chlamydia\$).ti,ab.

8 (chlamydia\$ adj5 urethritis).ti,ab.

9 exp Trichomonas vaginalis/

10 trichomon*.ti,ab.

11 exp Lymphogranuloma Venereum/

12 (lymphogranuloma adj5 venere\$).ti,ab.

13 (lymphogranuloma adj5 inguinal\$).ti,ab.

14 exp Neisseria gonorrhoeae/

15 exp Gonorrhea/

16 gonorrh\$.ti,ab.

17 (gonococ\$ adj5 urethritis).ti,ab.

18 exp Syphilis/

19 syphili\$.ti,ab.

20 chancre\$.ti,ab.

21 lues.ti,ab.

22 exp Treponema pallidum/

23 (treponema adj5 pallid\$).ti,ab.

24 (spirochaeta adj5 pallida).ti,ab.

25 (treponema adj5 reiterii).ti,ab.

261 or 2 or 3 or 4 or 5 or 6 or 7 or 8 or 9 or 10 or 11 or 12 or 13 or 14 or 15 or 16 or 17 or 18 or 19 or 20 or 21 or 22 or 23 or 24 or 25

27 (professional adj5 intervention\$).ti,ab.

28 (education $\$$ adj 5 material\$).ti,ab.

29 (education $\$$ adj 5 meeting\$).ti,ab.

30 (local adj5 consensus).ti,ab.

31 (education\$ adj5 visit\$).ti,ab.

32 (opinion adj5 leader\$).ti,ab.

33 (patient adj5 mediated adj5 intervention\$).ti,ab.

34 (feedback adj5 audit).ti,ab.

35 exp Reminder Systems/

36 reminder\$.ti,ab.

37 exp Marketing/

38 marketing.ti,ab.

39 (market adj5 research).ti,ab.

40 exp Mass Media/

41 (mass adj5 medium).ti,ab.

42 (communications adj5 media).ti,ab.

43 (mass adj5 media).ti,ab.

44 publicity.ti,ab.

45 (financial adj5 intervention\$).ti,ab.

46 (provider adj5 intervention\$).ti,ab.

47 exp Fee-for-Service Plans/

48 (medical adj5 fee\$).ti,ab.

49 'Fee-for-Service'.ti,ab.

Quality improvement interventions for improving the detection and management of curable sexually transmitted infections in primary 
50 (physician adj5 fee\$).ti,ab.

51 (physician adj5 payment).ti,ab.

52 exp Prepaid Health Plans/

53 (prepaid adj5 health).ti,ab.

54 (health adj5 insurance).ti,ab.

55 (medical adj5 insurance).ti,ab.

56 exp Capitation Fee/

57 capitation.ti,ab.

58 'provider salaried service'.ti,ab.

59 (salar\$ adj5 service\$).ti,ab.

60 exp Prospective Payment System/

61 (prospective adj5 payment).ti,ab.

62 (prospective adj5 reimbursement\$).ti,ab.

63 (adjustment adj5 discretionary).ti,ab.

64 exp Physician Incentive Plans/

65 (physician adj5 incentive\$).ti,ab.

66 (institution\$ adj5 incentive\$).ti,ab.

67 (provider adj5 incentive\$).ti,ab.

68 (provider adj5 grant).ti,ab.

69 (provider adj5 allowance).ti,ab.

70 (institution\$ adj5 grant).ti,ab.

71 (institution $\$$ adj 5 allowance).ti,ab.

72 (provider adj5 penalty).ti,ab.

73 (institution\$ adj5 penalty).ti,ab.

74 formulary.ti,ab.

75 (organisation\$ adj5 intervention\$).ti,ab.

76 (organization\$ adj5 intervention\$).ti,ab.

77 (provider adj5 orientated adj5 intervention\$).ti,ab.

78 (revision adj5 professional adj5 role\$).ti,ab.

79 (clinical adj5 multidisciplinary adj5 team\$).ti,ab.

80 (formal adj5 integration adj5 service\$).ti,ab.

81 (skill adj5 mix).ti,ab.

82 exp Continuity of Patient Care/

83 (patient adj5 care).ti,ab.

84 (continuity adj5 care).ti,ab.

85 exp Job Satisfaction/

86 (job adj5 satisfaction).ti,ab.

87 (work adj5 satisfaction).ti,ab.

88 (material adj5 reward\$).ti,ab.

89 (psychic adj5 reward\$).ti,ab.

90 (communication adj5 health adj5 professional\$).ti,ab.

91 ('case discussion'adj5 health adj5 professional\$).ti,ab.

92 (structural adj5 intervention\$).ti,ab.

93 (change\$ adj5 service adj5 delivery).ti,ab.

94 (change $\$$ adj 5 physical adj 5 structure $\$$ ).ti,ab.

95 (change\$ adj5 facilit\$).ti,ab.

96 (change $\$$ adj 5 equipment).ti,ab.

97 (change $\$$ adj5 medical adj5 records).ti,ab.

98 (change $\$$ adj5 scope adj5 benefit $\$$ ).ti,ab.

99 (change $\$$ adj5 nature adj5 benefit\$).ti,ab.

100 (change\$ adj5 scope adj5 service\$).ti,ab.

101 (change $\$$ adj5 nature adj5 service\$).ti,ab.

102 (quality adj5 monitor\$).ti,ab.

Quality improvement interventions for improving the detection and management of curable sexually transmitted infections in primary 
103 (Ownership adj5 hospital\$).ti,ab.

104 (accreditation adj5 hospital\$).ti,ab.

105 ('affiliation status' adj5 hospital\$).ti,ab.

106 (Ownership adj5 clinic).ti,ab.

107 (accreditation adj5 clinic).ti,ab.

108 ('affiliation status' adj5 clinic).ti,ab.

109 (staff adj5 organisation).ti,ab.

110 (staff adj5 organization).ti,ab.

111 (regulatory adj5 intervention\$).ti,ab.

112 (medical adj5 liability).ti,ab.

113 (patient adj5 complaint\$).ti,ab.

114 exp Peer Review/

115 'peer review'.ti,ab.

116 'peer supervision'.ti,ab.

117 exp Licensure/

118 licens\$.ti,ab.

119 exp Quality Improvement/

120 (quality adj5 improvement).ti,ab.

121 (quality adj5 management).ti,ab.

12227 or 28 or 29 or 30 or 31 or 32 or 33 or 34 or 35 or 36 or 37 or 38 or 39 or 40 or 41 or 42 or 43 or 44 or 45 or 46 or 47 or 48 or 49 or 50 or 51 or 52 or 53 or 54 or 55 or 56 or 57 or 58 or 59 or 60 or 61 or 62 or 63 or 64 or 65 or 66 or 67 or 68 or 69 or 70 or 71 or 72 or 73 or 74 or 75 or 76 or 77 or 78 or 79 or 80 or 81 or 82 or 83 or 84 or 85 or 86 or 87 or 88 or 89 or 90 or 91 or 92 or 93 or 94 or 95 or 96 or 97 or 98 or 99 or 100 or 101 or 102 or 103 or 104 or 105 or 106 or 107 or 108 or 109 or 110 or 111 or 112 or 113 or 114 or 115 or 116 or 117 or 118 or 119 or 120 or 121

12326 and 122

124 (randomized controlled trial or controlled clinical trial).pt. or randomized.ab. or placebo.ab. or clinical trials as topic.sh. or randomly.ab. or trial.ti.

125 exp animals/ not humans.sh.

126124 not 125

127 intervention?.ti. or (intervention? adj6 (clinician? or collaborat\$ or community or complex or DESIGN\$ or doctor? or educational or family doctor? or family physician? or family practitioner? or financial or GP or general practice? or hospital? or impact? or improv\$ or individuali?e? or individuali?ing or interdisciplin $\$$ or multicomponent or multi-component or multidisciplin $\$$ or multi-disciplin $\$$ or multifacet $\$$ or multi-facet $\$$ or multimodal $\$$ or multi-modal $\$$ or personali?e? or personali?ing or pharmacies or pharmacist? or pharmacy or physician? or practitioner? or prescrib $\$$ or prescription? or primary care or professional $\$$ or provider? or regulatory or regulatory or tailor $\$$ or target $\$$ or team $\$$ or usual care) $)$.ab.

128 (pre-intervention? or preintervention? or 'pre intervention?' or post-intervention? or postintervention? or 'post intervention?').ti,ab. 129 (hospital\$ or patient?).hw. and (study or studies or care or health\$ or practitioner? or provider? or physician? or nurse? or nursing or doctor?).ti,hw.

130 demonstration project?.ti,ab.

131 (pre-post or 'pre test $\$$ ' or pretest $\$$ or posttest $\$$ or 'post test $\$$ ' or (pre adj5 post)).ti,ab.

132 (pre-workshop or post-workshop or (before adj3 workshop) or (after adj3 workshop)).ti,ab.

133 trial.ti. or ((study adj3 aim?) or 'our study').ab.

134 (before adj10 (after or during)).ti,ab.

135 ('quasi-experiment\$' or quasiexperiment\$ or 'quasi random\$' or quasirandom\$ or 'quasi control\$' or quasicontrol\$ or ((quasi\$ or experimental) adj3 (method $\$$ or study or trial or design $\$))$ ).ti,ab,hw.

136 ('time series' adj2 interrupt\$).ti,ab,hw.

137 (time points adj3 (over or multiple or three or four or five or six or seven or eight or nine or ten or eleven or twelve or month\$ or hour? or day? or 'more than')).ab.

138 pilot.ti.

139 exp Pilot projects/

140 (clinical trial or controlled clinical trial or multicenter study).pt.

141 (multicentre or multicenter or multi-centre or multi-center).ti.

142 random\$.ti,ab. or controlled.ti.

Quality improvement interventions for improving the detection and management of curable sexually transmitted infections in primary 
143 (control adj3 (area or cohort? or compare? or condition or design or group? or intervention? or participant? or study)).ab. not (controlled clinical trial or randomized controlled trial).pt.

144 (control year? or experimental year? or (control period? or experimental period?)).ti,ab.

145 evaluation studies as topic/ or prospective studies/ or retrospective studies/

146 (utili?ation or programme or programmes).ti.

147 (during adj5 period).ti,ab.

148 ((strategy or strategies) adj2 (improv\$ or education\$)).ti,ab.

149 (purpose adj3 study).ab.

150 'comment on'.cm. or review.pt. or (review not 'peer review\$').ti. or randomized controlled trial.pt.

151 (rat or rats or cow or cows or chicken? or horse or horses or mice or mouse or bovine or animal?).ti,hw. or veterinar\$.ti,ab,hw.

152 exp animals/ not humans.sh.

153127 or 128 or 129 or 130 or 131 or 132 or 133 or 134 or 135 or 136 or 137 or 138 or 139 or 140 or 141 or 142 or 143 or 144 or 145 or 146 or 147 or 148 or 149

154150 or 151 or 152

155153 not 154

156123 and 155

157123 and 126

Note: the CENTRAL search strategy doesn't include the terms \#124 - \#157.

\section{EMBASE.com}

\#1. 'chlamydia trachomatis'/exp

\#2. chlamydia:ab,ti

\#3. chlamidia:ab,ti

\#4. 'chlamydiasis'/

\#5. chlamydiasis:ab,ti

\#6. chlamidi*:ab,ti

\#7. (urogenital NEAR/5 chlamydia*):ab,ti

\#8. (chlamydia\$ NEAR/5 urethritis):ab,ti

\#9. 'trichomonas vaginalis'/

\#10. trichomon*:ab,ti

\#11. 'lymphogranuloma venereum'/

\#12. (lymphogranuloma NEAR/5 venere*):ab,ti

\#13. (lymphogranuloma* NEAR/5 inguinal ${ }^{*}$ ):ab,ti

\#14. 'neisseria gonorrhoeae'/exp

\#15. 'gonorrhea'/exp

\#16. gonorrh*:ab,ti

\#17. (gonococ* NEAR/5 urethritis):ab,ti

\#18. 'syphilis'/exp

\#19. syphili*:ab,ti

\#20. chancre*:ab,ti

\#21. lues:ab,

\#22. 'treponema pallidum'/exp

\#23. (treponema NEAR/5 pallid*):ab,ti

\#24. (spirochaeta NEAR/5 pallida):ab,ti

\#25. (treponema NEAR/5 reiterii):ab,ti

\#26. OR/ \#1 - \#25

\#27. (professional NEAR/5 intervention*):ab,ti

\#28. (education* NEAR/5 material*):ab,ti

\#29. (education* NEAR/5 meeting*):ab,ti

\#30. (local NEAR/5 consensus):ab,ti

\#31. (education* NEAR/5 visit*):ab,

\#32. (opinion NEAR/5 leader*):ab,ti

\#33. (patient NEAR/5 mediated NEAR/5 intervention*):ab,ti

\#34. (feedback NEAR/5 audit):ab,ti

Quality improvement interventions for improving the detection and management of curable sexually transmitted infections in primary 
\#35. 'reminder system'/exp

\#36. reminder*:ab,ti

\#37. 'marketing'/exp

\#38. marketing:ab,ti

\#39. (market NEAR/5 research):ab,ti

\#40. 'mass medium'/exp

\#41. (mass NEAR/5 medium):ab,ti

\#42. (communications NEAR/5 media):ab,ti

\#43. (mass NEAR/5 media):ab,ti

\#44. publicity:ab,ti

\#45. (financial NEAR/5 intervention*):ab,ti

\#46. (provider NEAR/5 intervention*):ab,ti

\#47. 'medical fee'/exp

\#48. (medical NEAR/5 fee*):ab,ti

\#49. 'fee-for-service':ab,ti

\#50. (physician NEAR/5 fee*):ab,ti

\#51. (physician NEAR/5 payment):ab,ti

\#52. 'health insurance'/exp

\#53. (prepaid NEAR/5 health):ab,ti

\#54. (health NEAR/5 insurance):ab,ti

\#55. (medical NEAR/5 insurance):ab,ti

\#56. 'capitation fee'/exp

\#57. capitation:ab,ti

\#58. (provider NEAR/5 salar*):ab,ti

\#59. (salar* NEAR/5 service*):ab,ti

\#60. 'prospective payment'/exp

\#61. (prospective NEAR/5 payment):ab,ti

\#62. (prospective NEAR/5 reimbursement*):ab,ti

\#63. (adjustment NEAR/5 discretionary):ab,ti

\#64. 'personnel management'/exp

\#65. (physician NEAR/5 incentive*):ab,ti

\#66. (institution* NEAR/5 incentive*):ab,ti

\#67. (provider NEAR/5 incentive*):ab,ti

\#68. (provider NEAR/5 grant):ab,ti

\#69. (provider NEAR/5 allowance):ab,ti

\#70. (institution* NEAR/5 grant):ab,ti

\#71. (institution* NEAR/5 allowance):ab,ti

\#72. (provider NEAR/5 penalty):ab,ti

\#73. (institution* NEAR/5 penalty):ab,ti

\#74. formulary:ab,ti

\#75. (organization* NEAR/5 intervention*):ab,ti

\#76. (organization* NEAR/5 intervention*):ab,ti

\#77. (provider NEAR/5 orientated NEAR/5 intervention*):ab,ti

\#78. (revision NEAR/5 professional NEAR/5 role*):ab,ti

\#79. (clinical NEAR/5 multidisciplinary NEAR/5 team*):ab,ti

\#80. (formal NEAR/5 integration NEAR/5 service*):ab,ti

\#81. (skill NEAR/5 mix):ab,ti

\#82. 'patient care'/exp

\#83. (patient NEAR/5 care):ab,ti

\#84. (continuity NEAR/5 care):ab,ti

\#85. 'job satisfaction'/exp

\#86. (job NEAR/5 satisfaction):ab,ti

\#87. (work NEAR/5 satisfaction):ab,ti

Quality improvement interventions for improving the detection and management of curable sexually transmitted infections in primary 
\#88. (material NEAR/5 reward*):ab,ti

\#89. (psychic NEAR/5 reward*):ab,ti

\#90. (communication NEAR/5 health NEAR/5 professional*):ab,ti

\#91. ('case discussion' NEAR/5 health NEAR/5 professional*):ab,ti

\#92. (structural NEAR/5 intervention*):ab,ti

\#93. (change* NEAR/5 service NEAR/5 delivery):ab,ti

\#94. (change* NEAR/5 physical NEAR/5 structure*):ab,ti

\#95. (change* NEAR/5 facilit*):ab,ti

\#96. (change* NEAR/5 equipment):ab,ti

\#97. (change* NEAR/5 medical NEAR/5 records):ab,ti

\#98. (change* NEAR/5 scope NEAR/5 benefit*):ab,ti

\#99. (change* NEAR/5 nature NEAR/5 benefit ${ }^{*}$ ):ab,ti

\#100.(change* NEAR/5 scope NEAR/5 service*):ab,ti

\#101. (change* NEAR/5 nature NEAR/5 service*):ab,ti

\#102.(quality NEAR/5 monitor*):ab,ti

\#103.(ownership NEAR/5 hospital*):ab,ti

\#104.(accreditation NEAR/5 hospital*):ab,ti

\#105.('affiliation status' NEAR/5 hospital*):ab,ti

\#106.(ownership NEAR/5 clinic):ab,ti

\#107.(accreditation NEAR/5 clinic):ab,ti

\#108.('affiliation status' NEAR/5 clinic):ab,ti

\#109.(staff NEAR/5 organisation):ab,ti

\#110.(staff NEAR/5 organization):ab,ti

\#111.(regulatory NEAR/5 intervention*):ab,ti

\#112.(medical NEAR/5 liability):ab,ti

\#113.(patient NEAR/5 complaint*):ab,ti

\#114.'peer review'/exp

\#115.'peer review':ab,ti

\#116.'peer supervision':ab,ti

\#117.'licensing'/exp

\#118.licens*:ab,ti

\#119.'total quality management'/exp

\#120. (quality NEAR/5 improvement):ab,ti

\#121.(quality NEAR/5 management):ab,ti

\#122.OR/\#27 - \#121

\#123.'randomized controlled trial'/exp OR blind procedure'/exp OR 'double blind procedure'/exp OR 'crossover procedure'/exp OR random*:ab,ti OR placebo*:ab,ti OR allocat*:ab,ti OR crossover*:ab,ti OR 'cross over':ab,ti OR trial:ti OR (doubl* NEXT/1 blind*): $\mathrm{ab}, \mathrm{ti}$

\#124.'animal'/de OR 'animal experiment'/de OR 'nonhuman'/de NOT ('animal'/de OR 'animal experiment'/de OR 'nonhuman'/de AND 'human'/de)

\#125.\#123 NOT \#124

\#126. \#26 AND \#122

\#127.intervention*:ti OR (intervention* NEAR/5 (clinician* OR collaborat* OR community OR complex OR design* OR doctor* OR educational OR 'family doctor' OR 'family physician' OR 'family practitioner' OR financial OR 'gp' OR 'general practice' OR hospital OR impact* OR improve* OR individuali* OR interdisciplin* OR multicomponent OR 'multi-component' OR multidiscipline* OR 'multi-disciplin' OR multifacet* OR 'multi-facet*' OR multimodal* OR 'multi-modal*' OR personali* OR pharmacies OR pharmacist* OR pharmacy OR physician* OR practitioner* OR prescribe* OR prescription* OR 'primary care' OR professional* OR provider* OR regulatory OR regulatory OR tailor* OR target* OR team* OR 'usual care')):ab

\#128.'pre intervention*':ab,ti OR preintervention*:ab,ti OR 'pre intervention':ab,ti OR 'post intervention*':ab,ti OR postintervention*: ab,ti OR 'post intervention':ab,ti

\#129.hospital*:ti OR patient*:ti AND (study:ti OR studies:ti OR care:ti OR health*:ti OR practitioner*:ti OR provider*:ti OR physician*:ti OR nurse*:ti OR nursing:ti OR doctor*:ti)

\#130.'demonstration project*':ab,ti

Quality improvement interventions for improving the detection and management of curable sexually transmitted infections in primary 
\#131.'pre post':ab,ti OR 'pre test':ab,ti OR pretest*:ab,ti OR posttest*:ab,ti OR 'post test':ab,ti OR (pre NEAR/5 post):ab,ti \#132.'pre workshop':ab,ti OR 'post workshop':ab,ti OR (before NEAR/3 workshop):ab,ti OR (after NEAR/3 workshop):ab,ti \#133.trial:ti OR (study NEAR/3 aim*):ab OR 'our study':ab

\#134.(before NEAR/10 (after OR during)):ab,ti

\#135.'quasi-experiment':ab,ti OR quasiexperiment*:ab,ti OR 'quasi random':ab,ti OR quasirandom*:ab,ti OR 'quasi control':ab,ti OR quasicontrol*:ab,ti OR ((quasi* OR experimental) NEAR/3 (method* OR study OR trial OR design*)):ab,ti

\#136.('time series' NEAR/2 interrupt $\left.{ }^{*}\right): a b, t i$

\#137.time:ab AND (points NEAR/3 (over OR multiple OR three OR four OR five OR six OR seven OR eight OR nine OR ten OR eleven OR twelve OR month* OR hour* OR day* OR 'more than')):ab

\#138.pilot:ti

\#139.'pilot study'/exp

\#140.'clinical trial':ti OR 'controlled clinical trial':ti OR 'multicenter study':ti

\#141.multicentre:ti OR multicenter:ti OR 'multi-centre':ti OR 'multi-center':ti

\#142.random*:ab,ti OR controlled:ti

\#143. (control NEAR/3 (area OR cohort* OR compare* OR condition OR design OR group* OR intervention* OR participant* OR study)):ab NOT ('controlled clinical trial':ab,ti OR 'randomized controlled trial':ab,ti)

\#144.control:ab,ti AND year*:ab,ti experimental:ab,ti AND year*ab,ti OR 'control period*':ab,ti OR 'experimental period*':ab,ti

\#145.'prospective study'/de OR 'retrospective study'/de

\#146.utilization:ti OR programme:ti OR programmes:ti

\#147.(during NEAR/5 period):ab,ti

\#148.((strategy OR strategies) NEAR/2 (improv* OR education*)):ab,ti

\#149.(purpose NEAR/3 study):ab

\#150.'comment on':ab,ti OR 'review'/it OR (review:ti NOT 'peer review':ti) OR 'randomized controlled trial (topic)'/de

\#151.rat:ab,ti OR rats:ab,ti OR cow:ab,ti OR cows:ab,ti OR chicken*:ab,ti OR horse:ab,ti OR horses:ab,ti OR mice:ab,ti OR mouse:

ab,ti OR bovine:ab,ti OR animal*:ab,ti OR veterinary*:ab,ti

\#153 OR/.\#127-\#152

\#154.\#150 OR \#151 OR \#152

\#155.\#153 NOT \#154

\#156.\#126 AND \#155

\#157.\#126 AND \#155 AND [embase]/lim [non-RCT results]

\#158.\#125 AND \#126

\#159. \#125 AND \#126 AND [embase]/lim [RCT results]

LILACS (iAHx interface)

(mh:(“Sexually Transmitted Diseases”)) AND (ti:(“quality”)) OR (ab:(“quality”)) AND db:(“LILACS”)

WHO International Clinical Trials Registry Platform ICTRP portal

quality AND chlam* OR gonorrh* OR syphili* OR trichomon* OR venere* or sex infection*

ClinicalTrials.gov

quality AND "Sexually Transmitted Diseases” (by topic)

\section{CONTRIBUTIONSOFAUTHORS}

Barbara Nattabi, Alice Rumbold and Sajni Gudka drafted the protocol. James Ward commented on and suggested revisions to the protocol. All authors approved the final version of the protocol.

Quality improvement interventions for improving the detection and management of curable sexually transmitted infections in primary 


\section{DECLARATIONSOF INTEREST}

Alice Rumbold and James Ward were co-investigators of a completed trial that will be considered for inclusion in the review (the STI in remote communities: improved and enhanced primary health care trial (STRIVE) trial).

Authors of included studies will not be involved in assessing and extracting data from their own studies.

Barbara Nattabi: None known.

Sajni Gudka: None known.

\section{SOURCES OF SUPPORT}

\section{Internal sources}

- No sources of support supplied

\section{External sources}

- National Health and Medical Research Council, Australia.

Barbara Nattabi is supported by a NHMRC Early Career Research Fellowship and Alice Rumbold is supported by a NHMRC Career Development Research Fellowship 